\title{
Travel Planning Concept Taking Road Infrastructure Condition into Account
}

\author{
Marcin Staniek, Elżbieta Macioszek, Grzegorz Sierpiński \\ Silesian University of Technology, Faculty of Transport \\ Department of Transport System and Traffic Engineering \\ Krasińskiego 8 Street, 40-019 Katowice, Poland \\ marcin.staniek@polsl.pl; elzbieta.macioszek@polsl.pl; grzegorz.sierpinski@polsl.pl
}

\begin{abstract}
Pavement Management System data with a travel planner for purposes of planning of travel routes avoiding sections of roads and streets which do not conform with specific technical quality levels established under a procedure of the road infrastructure condition assessment. The solution proposed in the study comprises a highly advanced Intelligent Transport System which increases the efficiency of traffic planning and organisation in a city, referred to as urban mobility. Furthermore, the paper addresses methods applied to assess the road pavement condition in the Pavement Management System based on visual solutions of pavement condition assessment, including methods based on stereo visual imaging of the road pavement examined. Information and Communication Technologies have been discussed with regard to a mobile travel planner enabling route planning in any location by means of mobile devices. The article also provides a case study of travel route planning with reference to pre-set parameters describing technical condition of road infrastructure.
\end{abstract}

Keywords: transportation, planning, travel planner, road infrastructure, pavement management system

\section{Introduction}

The Intelligent Transport Systems (ITS) currently used in many cities all over the world are technologically advanced tool kits which encompass such fields of expertise as telecommunications, information technology and measuring, but also methods and technologies of transport management aimed at increasing efficiency of the transport system, safety of transport participants and protection of the environment, including people and natural resources. Pursuing the strategy of urban mobility of public space based on implementation of the ITS in a pre-defined territory ensures improved flow capacity of the network of streets as well as traffic safety. What follows is the increase of travelling comfort and improvement of traffic conditions experienced by users of private means of transport as well as those who travel by collective transport, but also by non-protected users of road infrastructure such as pedestrians or bikers. One may observe decreased travel times and energy consumption rates which translate into improved quality of natural environment through reduced emissions. Deployment of the ITS also entails reduction of costs involved in management of the road vehicle fleet and maintenance of road infrastructure, including repairs and overhauls. On a global scale, it augments the growth potential and economic benefits of the area where ITS-based solutions have been adopted.

Road infrastructure is the transport system's key component whose main function is transportation of people and goods. Properly designed, managed and maintained operational, it ensures high quality indices of transport processes, which is often impossible to attain without the support provided by the ITS. In order to ensure road traffic safety $[1,2]$ and suitable maintenance of road infrastructure, procedures of road infrastructure condition assessment [3] are deployed, based on which condition reports are drawn up. Databases and interactive maps of the road infrastructure condition are developed in consideration of individual types of defects as well as degrees of their severity. An analysis of such a set of data from a strategic perspective makes it possible to plan the budget for the city's road infrastructure maintenance, and when it stems from an operational point of view, it enables planning of the sections in need of immediate repairs or overhauls. Having assumed a specific time horizon, one can also project the degradation process and take individual remedies into account.

Besides ITS-based technologies, also ICT solutions making use of all communication media (Internet, wireless network, Bluetooth systems etc.) for data sending and processing are implemented in smart cities. What ICT also 
encompasses in this respect is information applications, mobile solutions, tools and complex IT systems enabling data processing and transfer on a higher level of abstraction than hardware. An example of application of such a technology may an expanded travel planner which enables searching for connections between multiple points in the given territory and the pre-defined transport network using features of mobile devices. The data required for travel planning and organisation, such as timetables, locations of vehicle rental service points, are rendered available by local authorities for the sake of urban mobility fostering and by collective transport operators as a means to assist users in travel planning. The connection search criteria implemented in commonly used travel planners are those of distance and time of travel from the starting point to the destination. Based on the distance criterion, the algorithm searches for the shortest route. What is identified by the algorithm as optimum with regard to the time criterion is the travel with the shortest time. The modes which one take into account in travel planning in the pre-set area of the transport network usually comprise walking, biking, private and collective transport.

Under the international project entitled "A platform to analyse and foster the use of Green Travelling options (GREEN TRAVELLING)" implemented as a part of the ERANET Transport III Future Travelling programme, a concept was proposed to utilise the data concerning the road infrastructure condition stored in the Pavement Management System for purposes of transport planning and organisation entailing limited traffic in the road infrastructure elements whose technical parameters do not conform with a pre-assumed quality level. Details of the design, implementation and operation of the travel planner known as GT Planner have been provided in papers [4][5].

\section{Evaluation of road infrastructure in the Pavement Management System}

The road pavement evaluation procedure requires inspection of the road infrastructure and definition of technical and operational parameters which describe it. The set of elements describing the road pavement condition includes: transverse profile, longitudinal profile, skid resistance, type and structure of cracks, surface distresses as well as condition and nature of previous repairs (e.g. pavement patches). State-of-the-art measuring solutions applied in road pavement diagnostics do not involve interference into the road structure and enable the diagnostic procedure to be conducted without restricting or closing traffic lanes subject to measurements [3]. Not only do they make the measurements possible, but simultaneously ensure a high level of accuracy of the pavement condition assessment procedure and use a comprehensive approach to identification of measures which describe it. The relevant procedure of road infrastructure inspection as a part of pavement diagnostics under the Polish conditions is described in the Guidelines for Application of Pavement Condition Diagnostics.

One of the most popular diagnostic methods applied for examination of road pavements involves using equipment based on digital image processing and recognition technologies. Such operations as filtering, extraction and matching of objects, thresholding of the image intensity function or road surface representation in a three-dimensional space allow for identification and keeping records of road pavement defects such as bumps, patches, chipping, deformation or cracks, including longitudinal, transverse interconnected and technological cracks. As far as visual methods of road pavement diagnostics are concerned, stereovision imaging is but a sample of the entire array of solutions discussed. The most fundamental solutions in this group are stereovision methods based on application of linear lighting or a projector displaying specific light patterns in the road assessment process. The defects being identified are described as deviations between the theoretical light information (for perfectly even surface) and the actual deformed light line or deformed pattern [6], [7]. The aforementioned reference papers describe an option of representing transverse and longitudinal irregularities as well pavement defects using the measure of cross-correlation of the extracted objects by the corner detection method. Authors of papers [8],[9] and [10] have proposed compilation of visual data received from a 2D camera with a onedimensional image delivered by a linear camera and a light pattern generated by a laser pointer. Such a measuring instrument used for assessment of condition of the micro- and macro-structure of road pavements has been described in paper [6]. In order to increase the accuracy of description of road infrastructure condition imaging maps, authors of publication [8],[9] have proposed that the visual identification system should be combined with a system of reference points determined by laser scanning. Making use of laser representation for identification of reference points is possible owing to triangulation techniques or the LIDAR solution, i.e. a rotating scanning device. 
Once the road inspection procedure is completed, data describing the road infrastructure condition are recorded in an integrated information system, namely in the Pavement Management System. Records of the data stored in the system's databases are maintained with the accuracy of $1 \mathrm{~m}$ of the route covered. Aggregating them in a specific scope for the assumed lengths of measurement sections makes it possible to assign an averaged pavement condition to linking sections considered crucial from the perspective of travel route planning by means of a standard road network description as a graph of connections in routing algorithms.

\section{Road infrastructure condition on GT Planner's transport network graph}

In order to integrate data concerning the road infrastructure condition retrieved from the Pavement Management System with GT Planner's transport network model, it was proposed that a graph of connections should be developed with an additional attribute, i.e. a technical parameter describing its condition. For that purpose, it was necessary to process the data from the road infrastructure condition assessment system and save them in a format supported by GT Planner. The additional attribute of pavement condition is taken into account in the heuristic function of the routing algorithm with reference to the pre-set optimisation criteria. Details of and opportunities arising from application of heuristic functions have been reviewed in publication [11].

On account of the structure of the graph of connections implemented in GT Planner, being a set of links as per the Open Street Map (OSM), the Pavement Management System data describing pavement condition in a $1 \mathrm{~m}$ resolution require combination and determination of an averaged value of the infrastructure condition analogical to OSM sections. An example of aggregation of sections covered by a description of the road infrastructure condition in the Pavement Management System to a structure of links has been illustrated in Fig. 1.

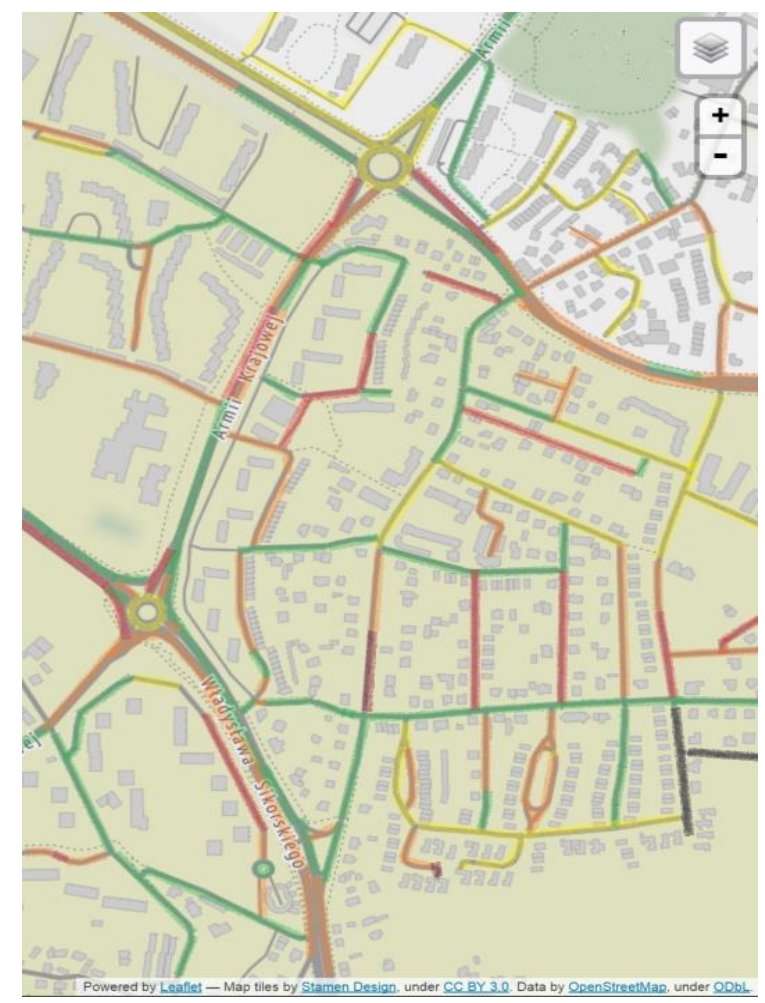

Fig. 1: Road infrastructure condition in the Pavement Management System. 


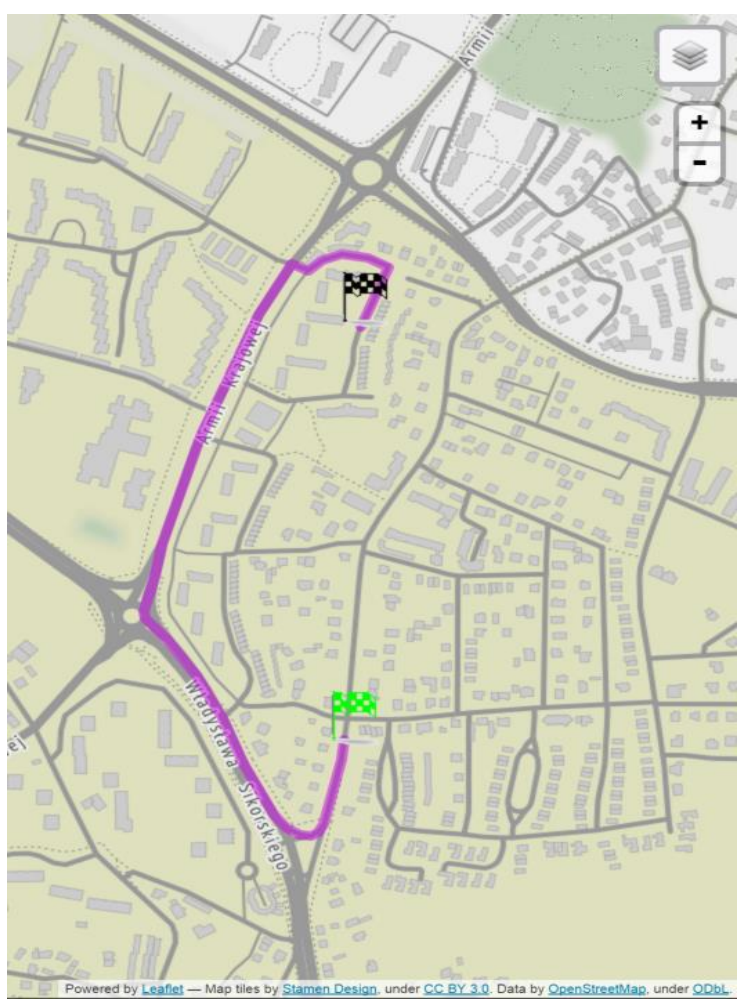

Fig. 2: Example of route planning taking road infrastructure condition into account.

Fig. 2 illustrates an example of route planning in GT Planner taking road infrastructure condition into account.

\section{Conclusions}

The concept of route planning by means of a mobile travel planner described in the paper allows for an optimum transfer route to be established taking the road infrastructure condition into account. From users' point of view, owing to this function, they can be certain about their travel proceeding in conditions of high comfort. For local authorities, on the other hand, route planning which takes restricted or closed road sections into account enables reducing the impact of vehicles on the technical condition of the infrastructure and performing the planned repairs or overhauls in the road network they manage.

\section{Acknowledgements}

The present research has been financed from the means of the National Centre for Research and Development as a part of the international project within the scope of ERANET Transport III Future Travelling Programme "A platform to analyze and foster the use of Green Travelling options (GREEN_TRAVELLING)".

\section{References}

[1] E. Macioszek, G. Sierpiński, L. Czapkowski, "Problems and Issues with Running the Cycle Traffic Through the Roundabouts," in Transport Systems Telematics. Communications in Computer and Information Science, Mikulski, Ed. Heidelberg Springer, vol. 104, pp. 107-114, 2010.

[2] T. Szczuraszek, E. Macioszek, "Proportion of Vehicles Moving Freely Depending on Traffic Volume and Proportion of Trucks and Buses," in The Baltic Journal of Road and Bridge Engineering, vol. 8, no 2, pp. 133-141, 2013.

[3] M. Staniek, "Stereo Vision Techniques in the Road Pavement Evaluation," in Proceedings of the XXVIII International Baltic Road Conference, Baltic Road Association, Vilnius, pp. 1-5, 2013.

[4] G. Sierpiński, M. Staniek, I. Celiński, "Travel behavior profiling using a trip planner," in Transportation Research Procedia, Elservier Amsterdam, vol. 14, 2352-1465, pp. 1743-1752, 2016. 
[5] G. Sierpiński, M. Staniek, I. Celiński, "Research And Shaping Transport Systems With Multimodal Travels Methodological Remarks Under The Green Travelling Project," in Proceedings of 7th International Conference of Education, Research and Innovation, Seville, pp. 3101-3107, 2014.

[6] A. Grace, D. Pycock, H. Tillotson, M. Snaith, "Active shape from stereo for highway inspection," in Machine Vision and Applications, vol. 12, no. 1, pp. 7-15, 2000.

[7] Y. Ma, S. Soatto, J. Kosecka, S. Sastry, An invitation to 3-D vision: from images to geometric models. SpringerVerlag, New York, 2004.

[8] C. Tsai, J. Wang, Y. Sun, "Livet stereoscopic visualization by using Hopfield neural nets," in Neural Computing and Applications, vol. 7, no. 3, pp. 229-237, 1998.

[9] J. Vilaça, J. Fonseca, A. Pinho, "Non-contact 3D acquisition system based on stereo vision and laser triangulation," in Machine Vision and Applications, vol. 21, no. 3, pp. 341-350, 2010.

[10] K. Wang, W. Gong, T. Tracy, V. Nguyen, "Automated survey of pavement distress based on 2D and 3D laser images," in Transportation Research Board, MBTC DOT 3023 - Grant, 2011.

[11] G. Sierpiński, I. Celiński, M. Staniek, "Using Trip Planners in Developing Proper Transportation Behavior," in International Journal of Social, Behavioral, Educational, Economic, Business and Industrial Engineering, World Academy of Science, Engineering and Technology, vol. 8, no. 11, 2014. 